\section{ETHICAL IMPLICATIONS OF BIOHACKING AS ACTIVISM: \\ DEMOCRATIZED \\ HEALTH CARE, DANGER, OR WHAT?}

\author{
JULIA ZHENG
}

\section{*}

\section{ABSTRACT}

Biohacking refers to optimizing one's body through modifying biology. In the $20^{\text {th }}$ century, do-ityourself (DIY) biology emerged as a type of biohacking involving biotechnology. Current highhealthcare costs promote DIY biology insulin and EpiPens as ways to challenge norms in healthcare, thus serving as forms of activism. Biohacked insulin is part of the \#WEARENOTWAITING movement to support improved treatment of Type 1 diabetes, whereas biohacked EpiPens allow people to make lifesaving autoinjectors at low costs. Social media acts as a catalyst and aids in the spread of insulin and EpiPen biohacking as activism. In 1979, Principles of Biomedical Ethics by Beauchamp and Childress proposed four principles that continue to guide decision-making in clinical medicine: beneficence, nonmaleficence, autonomy, and justice. This paper applies these principles to explore whether the benefits of performing DIY biology outweigh the potential health risks. Examining biohacking with a biomedical ethics frame, as outlined by Beauchamp and Childress, reveals that biohacking acts as a response to current issues but cannot serve as a solution in its current form. However, biohacking can grant patients more power in their relationship with the healthcare system, therefore lessening the dominance of formal institutions. Out of the four princi- ples, autonomy applies most differently when regarding biohacking than traditional medicine. Accordingly, a model of ethics for biohacking, such as of Beauchamp and Childress' with the autonomy altered to acknowledge the additional implications of biohacking, should be developed in the future.

\section{INTRODUCTION}

Recent rising healthcare costs and lack of insurance have jeopardized access to health care for many Americans. This problem has sparked a movement towards open-source medicine, leading to increased interest in biohacking. ${ }^{[5]}$ Biohacking is a broad term that refers to modifying one's biology in an informal setting. Examples of biohacking range from dieting to implanting computer chips in one's body. Specifically, do-it-yourself (DIY) biology is a type of biohacking that expands access to individuals, communities, and small organizations studying biology with the same biotechnology as formal research institutions. Biohacking has made biotechnology financially and intellectually accessible to those without proper training, who often work out of makeshift labs in their garages or kitchens. Pharmaceutical companies and the lucrative nature of the healthcare system have driven many Americans to take the issue into their own hands, forcing people to use DIY biology to synthesize their medicines or conduct genetic testing. Biohacking serves as an act of activism (which can be defined as campaigning to bring about change) against rising healthcare costs, providing otherwise powerless individuals a voice against the unfair practices of the healthcare system. Biohacking responds to high healthcare costs, but its viability from the perspective of biomedical ethics must be analyzed when determining if it can be a real solution to current issues.

Applying a biomedical ethics framework, like Beauchamp and Childress' prominent Principles of Biomedical Ethics, serves as a valuable context to explore the ethical implications of biohacking activism. The authors indicate four principles of biomedical ethics: beneficence, nonmaleficence, autonomy, and justice. These principles were first proposed in 1979 and are currently used widely by medical pro- 
fessionals and ethical review boards when making healthcare decisions. Beneficence refers to health care providers preventing harm and working to benefit the patient. Nonmaleficence holds healthcare professionals to a "standard of due care... taking sufficient and appropriate care to avoid causing harm, as the circumstances demand of a reasonable and prudent person," in which "reasonable" refers to one who approaches a situation with caution and sensibly takes action ${ }^{[1]}$. Nonmaleficence requires medical professionals to prevent harming patients purposefully or negligently. Autonomy allows patients the ability to make informed, voluntary decisions without controlling influences. Justice in healthcare refers to the equal treatment of patients, regardless of money, age, or race. Regarding biohacking, however, one must note that the biohacker is the patient and the medical professional, further complicating this issue.

This paper will apply the bioethical framework of Beauchamp and Childress to biohacked insulin and EpiPens to weigh the benefits and risks of biohacking as a potential solution to high costs imposed by pharmaceutical companies. First, it will discuss the ethical implications of biohacking activism concerning insulin for type 1 diabetes. Next, it will examine the DIY EpiPen as a form of biohacking activism for those with allergies. Then, it will compare autonomy in its traditional sense with autonomy in biomedical ethics. Continuing the discussion of autonomy, it will further explore its applications and nuances regarding parents and children before concluding with a proposed plan to promote future ethical activity.

\section{INSULIN ACTIVISM}

For patients with type 1 diabetes and those with type 2 diabetes who do not produce enough insulin, insulin therapy is necessary to keep blood sugar within a target range, prevent hypoglycemia and ketoacidosis, as well as ultimately sustain and enhance life. However, continuously rising insulin costs have presented a barrier to patients with diabetes from receiving this medication. In the past twenty years, the price of insulin has increased from $\$ 21$ per vial to $\$ 332$ per vial in 2019, a more than
1000\% increase. Dr. S. Vincent Rajkumar of the Mayo Clinic comments on this issue: "There is limited innovation when it comes to insulin; the more pressing

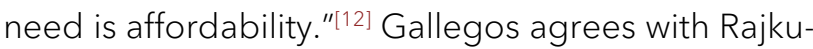
mar that these high costs present a concern and underscores instances of people "rationing treatments, using expired products, fasting, and even intentionally inducing diabetic ketoacidosis in order to obtain

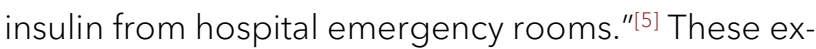
amples show that current healthcare conditions paradoxically push people to harm themselves in order to save their own lives. Beauchamp and Childress would view the issue presented by Rajkumar and Gallegos as a violation of the principle of justice, particularly distributive justice. ${ }^{[1]}$ Rajkumar agrees, stating that "Insulin pricing in the United States is the consequence of the exact opposite of a free market: extended monopoly on a lifesaving product in which prices can be increased at will..."[12] All four of these authors would argue that pharmaceutical companies behave unethically regarding the distribution of insulin, creating an environment that pushes people to engage in biohacking as activism.

Although insulin is not currently successfully synthesized using DIY biology, multiple organizations conduct experiments to move towards this goal. For instance, The Open Insulin Project is a collaboration of community biolabs worldwide that is currently attempting to produce insulin more cheaply than pharmaceutical companies. Once widespread, biohacked insulin could act as a life-saving alternative for patients who cannot afford traditional insulin. One main difference between formally produced and biohacked insulin is the rigorous testing a new drug must undergo in the United States, which "cost[s] between $\$ 30$ and $\$ 250$ million." Thus, regulatory costs create a major barrier to entry for potential biosimilar producers and necessitate high drug prices to recoup investments spent on clinical trials". ${ }^{[5]}$ Biohacked insulin can avoid extensive testing, but this lack of assurance results in risks of unintentional poisoning for consumers. The lack of required training for people to perform DIY biology also presents a risk. For instance, a biohacker working for the Open Insulin Project was described as "frequently check[ing] the instructions on his 
smartphone, because he is not too familiar with this kind of work yet...[he] has a major in business economics." ${ }^{\text {"[10] }}$ This behavior implies an unfamiliarity with the science, further highlighting the dangers of being unvetted and underprepared. Gallegos and Osterath demonstrate the trade-off between safety and financial accessibility that biohackers must consider. The problematic situation that people with diabetes find themselves in jeopardizes their ability to obtain a life-sustaining medication, resulting in desperation and fear of death that enables immense psychological discomfort. This situation prevents biohackers from acting as "reasonable" people and thus fully demonstrating nonmaleficence. Furthermore, trained professional workers in healthcare fulfill the principle of nonmaleficence by relying on their extensive training, following strict protocols, and practicing wise judgment to ensure nonmaleficence for their patients; this also includes obtaining medications and supplies from a trusted, formal source such as a pharmaceutical company. One could also argue that Beauchamp and Childress' "due care" refers to taking the proper, rather than biohacked, medication. Due to the lack of testing and regulation of DIY insulin in its current state, people cannot ensure the same degree of nonmaleficence as when taking a formally produced drug, presenting significant risks. On a small scale, those who synthesize and take biohacked insulin aim to benefit themselves, disqualifying these actions from serving as activism. However, the DIY synthesis of insulin, automated insulin delivery (AID) devices, and the spread of the movement using social media allow biohacking to serve as a form of activism and as a tool to promote change in diabetes treatment.

There is a distinction between type 1 and type 2 diabetes. Type 2 diabetes often develops due to lifestyle factors, whereas type 1 diabetes is mainly caused by genetics and typically affects people throughout their lives, starting from an early age. These conditions force type 1 diabetics to continuously rely on the healthcare system and create a vulnerability that allows pharmaceutical companies to capitalize on patients with no control over their lifelong disease. Frustration in the healthcare system and the status of diabetes treatment has led to the
\#WeAreNotWaiting movement, which consists primarily of type 1 diabetic individuals who advocate for increased access to insulin delivery technology through making their own AID devices. One advocate, Timothy Omer, has had type 1 diabetes for more than 22 years and currently works to develop a novel Artificial Pancreas System. He describes the reality of being a type 1 diabetic:

\footnotetext{
The most modern accessible technology for type 1 diabetes management is an insulin pump, which provides a constant supply of insulin, as well as a self-funded continuous glucose monitor, which provides real-time feedback of the patient's blood sugar levels. These devices provide many functions and high volumes of data, all of which are very welcome and useful, but such systems always fail with regards to patient expectations to understand and process all of this information. As a result, patients become overwhelmed by a feeling of judgement by healthcare professionals, the vast amounts of ensuing information, and alert and alarm "shouts" from their devices when they have failed at being a "good diabetic", as well as with their own disappointment of their body letting them down. ${ }^{[9]}$
}

Omer demonstrates that he and others are tired of waiting for a change in how type 1 diabetes treatment is approached. This frustration has pushed them to engage in biohacking in order to regain agency over their health condition. The physical act of making the AID device empowers patients to feel as though they are regaining power after years of feeling controlled by the healthcare system, and the low prices of this equipment make it increasingly financially accessible and allow it to act as a catalyst for the spread of the movement. One prime example is one mother's model of a DIY artificial pancreas for her daughter: "Sydney, now 15, is still using an updated version of that DIY system, which, because a fellow DIYer donated the pump, cost only $\$ 250$ to make...Apple Inc. and Eli Lilly \& Co. have hired DIYers, and Medtronic's latest FDA-approved product can now do most of the things the Farnsworths' system can-for $\$ 7,000$, before insurance..."[6] Not only is the act of making one's device (previously reserved for healthcare professionals) shocking, but the significantly lower prices allow the \#WEARENOTWAITING movement to draw media attention and gain support. Although only a tiny portion of the population lives with type 1 diabetes, almost 
everyone can relate to feeling frustrated or upset at high medical costs. Also, regardless of social class and safety factors, people would favor lower healthcare costs, making it easy for the \#WEARENotWaiting movement to draw support. Previously, pharmaceutical companies held power to impose high prices, knowing that patients would likely try to obtain these devices or medications at all costs. However, if biohacking continues to grow, it could provide an alternative way for people to obtain medicines, threatening the long-held domination of the healthcare system. This change would represent a revolution in the way people view healthcare, allowing biohacking insulin on a large scale to serve as activism. Further, biohacking not only democratizes medicine by making it increasingly financially accessible but also simplifies science so that people without advanced degrees can intellectually comprehend the processes, fulfilling Beauchamp and Childress' principle of distributive justice. They indicate: "distribution of all rights and responsibilities in society, including, for example, civil and political rights." ${ }^{[1]}$ Perhaps it is time to provide patients who suffer from these lifelong conditions, rather than the monopolistic healthcare system, a greater say in their treatment; biohacking offers them the opportunity to do so.

\section{DIY EPIPEN}

Although biohacking insulin and EpiPens are similar in that they are critical to the lives of those with diabetes and life-threatening allergies, EpiPens differ from insulin regarding cost, safety, and the main ethical principles that pertain to situations in which one requires these medications. Biohacking insulin requires the actual medicine to be synthesized while biohacking EpiPens does not. In contrast, only the injector is made for EpiPens. This cost difference would lead to varying levels of accessibility. For instance, synthesizing insulin requires not only chemicals that could prove unattainable for many but it also requires the intellectual and scientific knowledge and ability to synthesize this medication. This process can be contrasted with that of the DIY EpiPen, which can be made using "off-the-shelf" parts for as low as $\$ 30$, making the process intellec- tually more accessible to those without a scientific background. ${ }^{[4]}$ One prominent distinction between DIY insulin and EpiPen is that the epinephrine in a DIY EpiPen can still come from a formal supplier, resulting in differences regarding safety. While DIY insulin contains a risk of poisoning oneself, the use of formally produced epinephrine in DIY EpiPens reduces the risk of physical harm to individuals in comparison. Although diabetes and allergies are both chronic conditions, there is the distinction of EpiPens as necessary upon exposure to an allergen to prevent immediate death. Beauchamp and Childress highlight this concept in their principle of beneficence, which includes "Rescue persons in danger."[1] People with diabetes need insulin over time to prolong their lives, but the immediate necessity of an EpiPen upon exposure to an allergen makes the danger of being without the life-saving device more imminent. The low costs, intellectual accessibility, and relative safety of these DIY EpiPens combined with the inherent, natural compulsion for humans to help other humans in cases of immediate need promote the DIY EpiPen movement as a form of activism.

In 2016, the price increase in the Mylan EpiPen received media attention and backlash from consumers. The increasing prices had been an issue, increasing from "...\$103.50 for a set. By July 2013, the price was up to $\$ 264.50$, and it rose 75 percent to $\$ 461$ by last May. This May the price spiked again to $\$ 608.61 \ldots{ }^{\prime[11]}$ As a response to these prices, Four Thieves Vinegar, a biohacking group founded in 2015, posted a YouTube video demonstrating how to inject oneself with a homemade epinephrine auto-injector and published a list of materials on how to do so. Donovan states that "shortly after the release of the video, other biohacker groups and DIYers began offering epinephrine auto-injector alternatives online. ProgressTH (an international design lab) released a statement announcing the development of a 3D concept for an at-home alternative auto-injector, which would be printed for as little as $\$ 3$ in materials. "[2] Although these biohackers primarily publicize their methods to help those with lifethreatening allergies stay safe, they expose the healthcare industry's issues and demonstrate the 
dire need for change, qualifying them as accidental activists. The cost of biohacked EpiPens is meager compared to traditional EpiPen costs, ranging from $\$ 600$ to $\$ 700$; even those without financial strain would likely consider it an option to save money. However, these videos demonstrate how cheap the materials in the EpiPen are. Suppose an average person were to figure out a way to produce the injector as cheaply as $\$ 3$. In that case, corporations almost certainly have ways to make them even cheaper, exposing the inflated prices. Companies that manufacture these devices could charge less and still make a profit, but they choose not to as a way to capitalize on people's vulnerability. Instead, this selfishness forces people to risk harming themselves using a biohacked injector to save their lives. The shocking idea of producing one's own EpiPen resulted in the idea's spread through the media, which would only result in positive reinforcement for the movement's growth. Increasing numbers of posts and videos on the media pertaining to DIY EpiPens normalizes the concept and reduces its stigma. As a result, more people are likely to try to make their own EpiPens, further propagating the movement. For the rest of society, this movement's media attention raises the question of what other medications corporations are overcharging for, sparking frustration against the healthcare industry, and fueling a more significant activist movement.

In financial terms, saving money through producing one's EpiPens would prevent a person from potentially overspending beyond his or her resources, fitting under the bioethical principle of beneficence. Beauchamp and Childress also emphasize the physical aspects of beneficence:

\footnotetext{
... a person $X$ has a determinate obligation of beneficence towards person $Y$ if and only if each of the following conditions is satisfied (assuming $X$ is aware of the relevant facts):

1. $Y$ is at risk of significant loss of or damage to life or health or some other major interest.

2. X's action is needed (singly or in concert with others) to prevent this loss or damage.

3. X's action (singly or in concert with others) has a high probability of preventing it.

4. X's action would not present significant risks, costs, or burdens to $X$.

5. The benefit that $Y$ can be expected to gain outweighs any harms, costs, or burdens that $X$ is likely to incur. ${ }^{[1]}$
}

In biohacking, however, $X$ and $Y$ would usually be the same person, and in certain instances, a parent and child (a concept that I will later explore). Number 1 is satisfied by a person being in anaphylactic shock, and number 2 represents an EpiPen's nature. Yet, the other points must be examined. Regarding numbers 3 and 5, one could argue that DIY epinephrine injectors have high risks compared to those formally produced. For instance, Willingham states, "A syringe doesn't offer the benefit and safety advantage of a well-calibrated dose, and it carries the risk of injection into a vein, instead of muscle, which can be fatal." With an untrained individual administering a medical technique that he or she does not have formal training in, there are risks. Yet, the necessary medication must be administered to patients with diabetes or allergies in the case of imminent death. Strikingly, the fourth idea is one of the main benefits of biohacking. For one person, the cost of EpiPens for her son was "more than her mortgage payment," and "her older son...just carries around expired EpiPens." "[13] Parents are forced to make the difficult decision of risking their child's life or overstretching their financial needs, and biohacking allows them to prevent both. The high and unattainable costs of insulin and EpiPens create desperation in patients, resulting in the sense of hopelessness that prices will become unattainable and ultimately an overwhelming fear of death without the medication. Desperation often pushes people to do things they would not otherwise consider, such as synthesizing their own medicines. Biohacking serves as a form of activism: while trying to save their own lives, biohackers also promote change in the way people view the severity of high healthcare costs. $\mathrm{Bi}$ ohacking's attention, especially when spread using social media, allows others to support the movement and promote social and economic change, qualifying biohackers as activists. In the hands of the healthcare system, people often have no choice but to follow the rules and policies put in place. However, biohacking allows these individuals to act and change the status quo. Therefore, biohacking serves as a physical representation of the changes people have wanted to make in the healthcare system. Although the act of biohacking does not directly result 
in fair healthcare practices and prices, perhaps its shocking nature is enough to generate attention and support to move towards change.

\section{AUTONOMY: TWO DIFFERENT TYPES?}

Autonomy, among Beauchamp and Chil-

dress' four principles of biomedical ethics, requires more examination due to the distinction between the traditional definition of autonomy and medical autonomy. Biohackers' ability to synthesize their insulin and EpiPens grants them a powerful sense of independence that they did not previously have; they are autonomous in its traditional sense, meaning independent. However, Beauchamp and Childress identify medical autonomy: "... in terms of normal choosers who act (1) intentionally, (2) with understanding, and (3) without controlling influences that determine their action... a broad continuum exists on which autonomy goes from being fully present to being wholly absent." ${ }^{[1]}$ In medicine, autonomy must involve the patient demonstrating understanding of a treatment or procedure, and medical professionals must adequately inform their patients. However, when people become biohackers due to desperation, they face no obligation to understand the risks of their actions thoroughly. Without being medically autonomous, biohackers are inhibited from acting ethically when examined with Beauchamp and Childress' model. The nature of biohacking facilitates impulsivity: "The ways in which DIY biology is 'governed' or 'regulated' takes a distinctive form: rather than being top-down it is bottom-up; rather than being defined by institutions or policymakers, it is collectively and openly negotiated by a large group of people..." ${ }^{[7]}$ Because biohacking relies on the public to regulate themselves rather than formal rules, it can be easily misused, proving extremely dangerous. On the other hand, though, many advancements in science have begun with experimentation, leading to ideas that may take many years for the public to accept. Perhaps biohacking is just one of these ideas that currently seems outlandish. As the concept continues to gain momentum and more safety measures are enacted, biohacking can be the next stage of scientific development.

\section{APPLICATIONS OF AUTONOMY}

The high costs of necessary technology combined with the monopolistic nature of diabetic health care can cause people to feel powerless at the hands of the healthcare system. As a result, making their own technology grants them a feeling of financial and personal autonomy. Currently, there is little to no formal regulation preventing this self-experimentation. Although there are no proper laws against biohacking insulin devices in the United States, the German model serves as an example of the current regulations: "Healthcare professionals must point out the dangers that may arise when using a DIY AID system and should clearly distance themselves from the use of an open system and not encourage patients to use the system. ${ }^{.[8]}$ Of course, healthcare professionals are worried about their patients, but they also benefit from higher medications and treatment costs. Healthcare professionals fail to advocate for cheaper alternatives, leaving patients with no one to fight for them. Patients must engage in activism to promote the change they want, and biohacking is one way for them to do so. The lack of limitation from governments, healthcare professionals, and finances grants patients autonomy from a biomedical ethics perspective. Many patients who feel "overwhelmed" by the health care system are now free from this feeling. Beauchamp and Childress state: "We encounter many problems of autonomy in medical contexts because of the patient's dependent condition and the medical professional's authoritative position... In these instances, the patient's autonomy may be compromised because the physician has assumed an unwarranted degree of authority over his or her patient."[1] Not only do health care professionals hold authority over patients under the traditional health care system, but the healthcare industry controls the price and distribution of insulin and devices. However, health care professionals lack the right to prevent patients from making and using DIY AID systems. Therefore, when juxtaposed with the legal inability of healthcare professionals to prevent patients from using DIY biology to create their AID systems, patients are now free from what they feel is domination by the health care system. 


\section{IF INCREASINGLY ACCEPTED BY THE PUBLIC, BIOHACKING CAN DRASTICALLY CHANGE THE WAY PEOPLE VIEW THE RELATIONSHIP BETWEEN PATIENTS AND THE HEALTHCARE SYSTEM.}

\section{6}

AUTONOMY OF PARENTS \& CHILDREN

The issue of people using biohacking on themselves is less controversial than people performing biohacking on others. Specifically, the case of parents using biohacked medicine and devices on their children sparks controversy. Because the children are dependent, they do not have the authority to refuse their parents nor the resources to obtain the medication for themselves through formal means. The extent to which parents must follow the principles of biomedical ethics must be examined. For instance, Beauchamp and Childress identify negligence as a subcategory of nonmaleficence: "Negligence is the absence of due care. In the professions, it involves a departure from the professional standards that determine due care in a given set of circumstances. The term negligence covers two types of situations: (1) intentionally imposing risks of harm that are unreasonable (advertent negligence or recklessness) and (2) unintentionally, but carelessly, imposing risks of harm (inadvertent negligence)." "[1] If parents do not attempt to obtain the insulin or EpiPen their child needs through formal means or biohacking, this could be regarded as negligent. Just as medical professionals are required to follow the widely agreed-upon rules and procedures to ensure the safety of their patients, a parallel can be drawn to the parents, in which they are expected to provide their children with the safest medications possible-those produced by formal institutions. Therefore, parents synthesizing pharmaceuticals using biohacking could also be considered negligent, where "due care" includes obtaining official medicine. The barriers to this situation should not be disregarded. Perhaps it is the pharmaceutical or insurance companies that are fostering this negligence, and they should therefore bear more responsibility. Biohacking also introduces an additional risk of harm for the children. For instance, the German laws for DIY AID systems indicate that "People who build DIY AID systems and transfer them to other patients are liable to prosecution under the Medical Devices Act in Germany. The placing on the market and commissioning of such a system are prohibited. The person who builds and transfers the system is responsible under the Product Liability Act. ${ }^{[8]}$ When biohacking for their children, parents take on additional ethical responsibility that, not being healthcare professionals, they may not be able to uphold. These parents likely never wanted this responsibility, but healthcare conditions forced them to act. These drastic measures show the public how dire the situation is and characterize these parents as activists.

\section{CONCLUSION}

The spread of biohacking techniques through social media and the internet can alter science and medicine, resulting in biohacking serving as a form of activism. One primary example of change brought about after the increase in biohacking is the reduced costs of the EpiPen, including the "release of a generic version of its device at about half the cost (about \$340) of the brand name EpiPen...CVS also announced that the manufacturer would provide a $\$ 100$ coupon for much of its financially insecure population to reduce the out-ofpocket cost to about $\$ 10$ per prescription. ${ }^{\prime[2]}$ It is possible that the media attention received by biohackers threatened pharmaceutical companies, as these companies feared losing profit as more people attempted to biohack. Nevertheless, the biohackers who spearheaded the DIY EpiPen movement achieved social and economic change, although they risked their lives in the process, representing activism. Perhaps these corporations will, in the future, be wary before imposing high and unattainable prices on consumers for fear of driving more people to biohack. If increasingly accepted by 
the public, biohacking can drastically change the way people view the relationship between patients and the healthcare system. Dyson proposes the future of biohacking:

There will be do-it-yourself kits for gardeners who will use genetic engineering to breed new varieties of roses and orchids. Also kits for lovers of pigeons and parrots and lizards and snakes to breed new varieties of pets. Breeders of dogs and cats will have their kits too... Few of the new creations will be masterpieces, but a great many will bring joy to their creators and variety to our fauna and flora. The final step in the domestication of biotechnology will be biotech games, designed like computer games for children down to kindergarten age but played with real eggs and seeds rather than with images on a screen. Playing such games, kids will acquire an intimate feeling for the organisms that they are growing. The winner could be the kid whose seed grows the prickliest cactus, or the kid whose egg hatches the cutest dinosaur. ${ }^{[3]}$

Dyson emphasizes the idea that biohacking will "bring joy" and that eventually, people will perform biohacking for pleasure, in contrast to something that is currently performed out of desperation.

Although biohacking is still a new topic, the media attention it receives (coupled with its low costs) can lead to the democratization of healthcare and science. In its current state, biohacking presents a tempting option for those who seek to avoid the high costs of the traditional healthcare system. Yet, the risks of this unregulated experimentation deter many rationally thinking people. However, in the future, if ethical and safety regulations are put in place, people begin devising new ways to use biohacking and posting these ideas on the media; biohacking can break financial and intellectual bounds. Although the first amendment protects free speech, sites can employ community rules or rules of conduct to promote the safety of biohacking. No longer would people feel helpless under a system that controls their health. If perfected and made applicable to more situations, biohacking could challenge the long-standing dominance of formal institutions and grant more power to the average person. In activism, people often seek the traditional definition of justice, which typically means fairness. Biohackers fall under this category and aim to achieve fair prices and access to medications. However, just as there are multiple definitions of autonomy, there are also different forms of justice; Beauchamp and Childress define the concept of justice in a biomedical ethics context slightly differently. They describe their idea of material justice: "...primarily our obligations are limited to fundamental needs. To say that someone has a fundamental need is to say that the person will be harmed or detrimentally affected in a fundamental way if that need is not fulfilled." [1] Biohacking finally allows ordinary people to bring attention to the message that their needs are not met. Although this purpose was likely not intentional, by democratically synthesizing one's medication, biohackers also fulfill the idea of justice from a biomedical ethics perspective, aside from simply seeking justice in its traditional sense.

The idea of biohacking, or performing experiments once only reserved to formal institutions, can be shocking and disturbing to many. Biohacking has the potential to go wrong and harm people. Yet, in the case of chronic medical conditions such as diabetes and allergies, biohacking offers a sense of control and a glimmer of hope for those put in a challenging financial and medical situation. When examining types of activism from rallies to boycotts to hunger strikes, all of these examples share one common feature with biohacking: they are striking to the public. Real, lasting change rarely comes from mundane activities, so perhaps the dangerous nature of biohacking is critical to it serving as activism. Also, low costs and media attention aid in biohacking spreading. When examining these applications of biomedical ethics using Beauchamp and Childress' model, many nuances prevent biohacking from serving as a straightforward solution to high healthcare costs. However, because their principles apply to traditional medicine rather than biohacked treatments, these principles should be updated to reflect the ethical concerns over the increasing popularity of biohacking. Without an exact code of instructions, biohackers are left to their own devices. Although biohackers have developed their code of ethics consisting of broad principles such as responsibility and transparency, this unofficial code resembles more of a list than rules for biohackers to follow. When juxtaposed with Beauchamp and Childress' model, the biohacker code of ethics is concise and nonspecific. 
Therefore, perhaps it is time to develop a model of ethics for biohackers. Out of the four principles, beneficence, nonmaleficence, and justice can remain largely the same. However, due to the freedom granted by individuals performing biohacking on themselves, the principle of autonomy should be altered to emphasize the importance of a complete understanding of risks and rewards before one performs biohacking to ensure its ethical soundness as increasing numbers of people engage in biohacking in hopes of saving lives and promoting change

\section{REFERENCES}

[1] Beauchamp, Tom L., and Childress, James L. Principles of Biomedical Ethics. $8^{\text {th }}$ ed. Oxford University Press, 2019.

[2] Donovan, Matthew C. J. Pop-up Maktivism: A Case Study of Organizational, Pharmaceutical, and Biohacker Narratives. 2019. Arizona State University. PhD dissertation.

[3] Dyson, Freeman. "Our Biotech Future." New York Review of Books, vol. 54, no, 12, 2007.

[4] Four Thieves Vinegar, accessed: 6 Dec. 2020, HTTPS://WWW.FOURTHIEVESVINEGAR.ORG

[5] Gallegos, Jenna E., et al. "The Open Insulin Project: A Case Study for 'Biohacked' Medicines." Trends in Biotechnology, vol. 36, no. 12, 2018, pp. 1211-1218.

[6] Kresge, Naomi and Cortez, Michelle. "The $\$ 250$ Biohack That's Revolutionizing Life With Diabetes." Bloomberg, 2018.

[7] Landrain, Thomas et al. "Do-it-yourself biology: challenges and promises for an open science and technology movement." Systems of Synthetic Biology, vol. 7, no. 3, 2013, pp. 115-126.

[8] Oliver, Nick et al. "Open source automated insulin delivery: addressing the challenge." Digital Medicine, no. 124, 2019.

[9] Omer, Timothy. "Empowered Citizen 'Health Hackers' Who are Not Waiting." BMC Medicine, vol. 14, no. 118, 2016.

[10] Osterath, Brigitte. "Do-It-Yourself Insulin: Biohackers Aim to Counteract Skyrocketing Prices." DW, 2019, WWW.DW.COM/EN/DO-IT-YOURSELF-INSULIN-BIOHACKERS-AIM-TOCOUNTERACT-SKYROCKETING-PRICES/A-48861257.

[11] Parker-Pope, Tara and Peachman, Rachel Rabkin. "EpiPen Price Rise Sparks Concern for Allergy Sufferers." The New York Times, 2016.

HTTPS://WELL.BLOGS.NYTIMES.COM/2016/08/22/EPIPEN-PRICE-RISE-SPARKSCONCERN-FOR-ALLERGY-SUFFERERS

[12] Rajkumar, S. Vincent. "The High Cost of Insulin in the United States: An Urgent Call to Action." Mayo Clinic Proceedings, vol. 95, no. 1, 2020.

[13] Willingham, Emily. "Why Did Mylan Hike EpiPen Prices 400? Because They Could." Forbes, 2016. HTTPS://WWW.FORBES.COM/SITES/EMILYWILLINGHAM/2016/08/21/WHYDID-MYLAN-HIKE-EPIPEN-PRICES-400-BECAUSE-THEY-COULD/

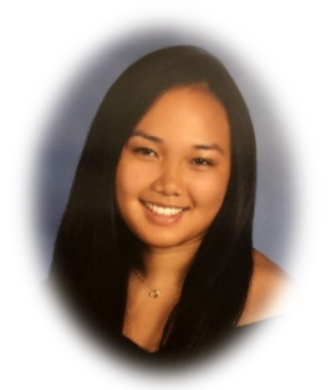

Julia Zheng is a junior at Rutgers University-New Brunswick pursuing a Bachelor of Science in Cell Biology and Neuroscience in the School of Arts and Sciences Honors Program with a minor in Psychology. She conducted research for this paper in her Research in the Disciplines: Science, Medicine, and Society course in Fall 2020. Julia is interested in science and medicine and has the goal of pursuing a career in the medical field as a physician. At Rutgers, Julia is President of the Bioethics Society, conducts research in Dr. Alexander Kusnecov's lab, and works as a part-time lecturer for the Physics Department. Outside of school, she works as a medical assistant and receptionist at a pediatrician's office and enjoys volunteering in her community. 